Tomasz SuŁKowski (Zielona Góra)

\title{
MATHEMATICAL DESCRIPTION OF THE PHASE TRANSITION CURVE NEAR THE CRITICAL POINT
}

Abstract. In this paper, by applying a simple mathematical model imitating the equation of state, behaviour of the phase transition curve near the critical point is investigated. The problem of finding the unique vapour-liquid equilibrium curve passing through the critical point is reduced to solving a nonlinear system of differential equations.

1. Introduction. For chemical engineering applications it is necessary to predict very accurately the vapour-liquid equilibrium (VLE) curve (see $[1,2])$. Before the more detailed formulation of the problem we introduce some important quantities in thermodynamics of fluids. Let $\varrho, T$ and $P$ denote the density, temperature and pressure of the fluid, respectively. We assume that the equation of state (EOS) is given by $P=P(\varrho, T)$, where $P$ is an analytic function of $T$ and $\varrho$. Let $\mu=\mu(\varrho, T)$, an analytic function of $T$ and $\varrho$, denote the chemical potential of the fluid. We can write the basic thermodynamic relation between $P$ and $\mu$ as follows:

$$
\frac{\partial \mu}{\partial \varrho}=\frac{1}{\varrho} \frac{\partial P}{\partial \varrho} .
$$

The VLE curve is given by two curves $\varrho_{V}=\varrho_{V}(T)$ and $\varrho_{L}=\varrho_{L}(T)$, which are defined for any $T$ below the critical temperature $T_{C}$. These curves satisfy the classical thermodynamic requirements (see $[1,2]$ )

$$
\left\{\begin{array}{l}
P\left(\varrho_{V}(T), T\right)=P\left(\varrho_{L}(T), T\right), \\
\mu\left(\varrho_{V}(T), T\right)=\mu\left(\varrho_{L}(T), T\right) .
\end{array}\right.
$$

Moreover, $\varrho_{V}(T)<\varrho_{L}(T)$ for $T<T_{C}$ and $\varrho_{V}\left(T_{C}\right)=\varrho_{L}\left(T_{C}\right)=\varrho_{C}$, where $\varrho_{C}$ is called the critical density. The critical temperature and critical density

2000 Mathematics Subject Classification: 34A34, 34C60, 80A17.

Key words and phrases: VLE curve, EOS, nonlinear differential equations. 
determine the so-called critical point. It is known that at the critical point

$$
\dot{\varrho}_{V}\left(T_{C}\right)=\infty \text { and } \dot{\varrho}_{L}\left(T_{C}\right)=-\infty .
$$

By physical considerations we also have

$$
\frac{\partial P}{\partial \varrho}\left(\varrho_{V}(T), T\right) \neq 0 \quad \text { and } \quad \frac{\partial P}{\partial \varrho}\left(\varrho_{L}(T), T\right) \neq 0 .
$$

The determination of the critical point from the thermodynamic properties of the fluid is one of the main efforts of chemical engineering.

For each given temperature $T<T_{C}$, the system of equations (2) must be solved. Recently a new, easier and quicker, method has been proposed to solve this system (see $[4,5]$ ). Practically, this method gives us a continuous mathematical model for $\varrho_{V}=\varrho_{V}(T)$ and $\varrho_{L}=\varrho_{L}(T)$, without having to solve (2) point by point. The method is based on solving a system of nonlinear differential equations and can be applied to every known EOS. It is almost impossible to solve this system analytically, therefore numerical methods should be used. However, some examples show that small changes of initial conditions for the system of differential equations imply that finding the VLE curve is impossible because the numerically determined $\varrho_{V}$ and $\varrho_{L}$ cannot cross at the critical point (see $[4,5]$ ).

In this paper we will construct a simple mathematical model imitating the equation of state and we will try to explain the above-mentioned difficulties. On the basis of this model we shall show that there exist unique initial conditions $\varrho_{V}^{0}=\varrho_{V}\left(T_{0}\right), \varrho_{L}^{0}=\varrho_{L}\left(T_{0}\right)$ for fixed $T_{0}<T_{C}$, such that the system of differential equations has a unique solution $\left(\varrho_{V}(T), \varrho_{L}(T)\right)$ passing through the critical point.

2. Theoretical background of the method. In this part we recall the theoretical base of the method presented in $[4,5]$.

For any $T$ below the critical temperature $T_{C}$, the curves $\varrho_{V}=\varrho_{V}(T)$ and $\varrho_{L}=\varrho_{L}(T)$ satisfy (2). Since $P$ and $\mu$ are given by analytical formulas, differentiating the equations (2) with respect to $T$ we get

$$
\left\{\begin{array}{l}
\frac{\partial P}{\partial T}\left(\varrho_{V}(T), T\right)+\frac{\partial P}{\partial \varrho}\left(\varrho_{V}(T), T\right) \dot{\varrho}_{V}=\frac{\partial P}{\partial T}\left(\varrho_{L}(T), T\right)+\frac{\partial P}{\partial \varrho}\left(\varrho_{L}(T), T\right) \dot{\varrho}_{L}, \\
\frac{\partial \mu}{\partial T}\left(\varrho_{V}(T), T\right)+\frac{\partial \mu}{\partial \varrho}\left(\varrho_{V}(T), T\right) \dot{\varrho}_{V}=\frac{\partial \mu}{\partial T}\left(\varrho_{L}(T), T\right)+\frac{\partial \mu}{\partial \varrho}\left(\varrho_{L}(T), T\right) \dot{\varrho}_{L}
\end{array}\right.
$$

where $\dot{\varrho}_{V}$ and $\dot{\varrho}_{L}$ denote the derivatives of $\varrho_{V}$ and $\varrho_{L}$ with respect to $T$.

From (3) we obtain the system of two nonlinear differential equations of first order

$$
\left\{\begin{array}{l}
\dot{\varrho}_{V}=f\left(\varrho_{V}, \varrho_{L}, T\right) \\
\dot{\varrho}_{L}=f\left(\varrho_{L}, \varrho_{V}, T\right)
\end{array}\right.
$$


where

$$
\begin{aligned}
f\left(\varrho_{V}, \varrho_{L}, T\right) \\
\quad=\frac{\left(\frac{\partial \mu}{\partial T}\left(\varrho_{V}, T\right)-\frac{\partial \mu}{\partial T}\left(\varrho_{L}, T\right)\right) \varrho_{L} \varrho_{V}+\left(\frac{\partial P}{\partial T}\left(\varrho_{L}, T\right)-\frac{\partial P}{\partial T}\left(\varrho_{V}, T\right)\right) \varrho_{V}}{\frac{\partial P}{\partial \varrho}\left(\varrho_{V}, T\right)\left(\varrho_{V}-\varrho_{L}\right)} .
\end{aligned}
$$

Since the right-hand side of (5) is sufficiently regular, the system (4) has unique solutions $\varrho_{V}$ and $\varrho_{L}$ for given $\varrho_{V}^{0}=\varrho_{V}\left(T_{0}\right)$ and $\varrho_{L}^{0}=\varrho_{L}\left(T_{0}\right)$, where $T_{0}<T_{C}$ (see [3]). Obviously, it is almost impossible to find analytic expressions for solutions of (4) for given $P$ and $\mu$.

3. Simple mathematical model imitating the equation of state (EOS). It is well known that the function $P=P(\varrho, T)$ has the following properties:

- $P(0, T)=0$ for any $T$;

- for fixed $T<T_{C}$ the function $P$ has two extrema, a maximum and a minimum;

- for fixed $T>T_{C}$ the function $P$ is strictly increasing with respect to $\varrho$;

- $\frac{\partial P}{\partial \varrho}\left(\varrho_{C}, T_{C}\right)=\frac{\partial^{2} P}{\partial^{2} \varrho}\left(\varrho_{C}, T_{C}\right)=0$;

- some observations suggest that for fixed $T$ the function $P(\varrho, T)$ is approximately cubic with respect to $\varrho$.

Having all this in mind we can model the qualitative properties of the function $P$ by the following formula:

$$
P(\varrho, T)=\varrho^{3}-3 \varrho^{2} \varrho_{C}+3 \varrho \varrho_{C}^{2}+\varrho\left(T-T_{C}\right),
$$

which behaves similarly to the Van der Waals equation of state near the critical point.

By (1) the function $\mu$ has the form

$$
\mu(\varrho, T)=\frac{3}{2} \varrho^{2}-6 \varrho \varrho_{C}+\left(3 \varrho_{C}^{2}+T-T_{C}\right) \ln \varrho+C(T) .
$$

Without loss of generality we can assume $T_{C}=0$.

Substituting (6) and (7) into (5) we get the system of two nonlinear differential equations

$$
\left\{\begin{array}{l}
\varrho_{V}=\frac{\varrho_{V} \varrho_{L} \ln \frac{\varrho_{V}}{\varrho_{L}}+\varrho_{V}\left(\varrho_{L}-\varrho_{V}\right)}{\left(3\left(\varrho_{V}-\varrho_{C}\right)^{2}+T\right)\left(\varrho_{V}-\varrho_{L}\right)}, \\
\dot{\varrho}_{L}=\frac{\varrho_{L} \varrho_{V} \ln \frac{\varrho_{V}}{\varrho_{L}}+\varrho_{L}\left(\varrho_{L}-\varrho_{V}\right)}{\left(3\left(\varrho_{L}-\varrho_{C}\right)^{2}+T\right)\left(\varrho_{V}-\varrho_{L}\right)} .
\end{array}\right.
$$

Since we consider $\varrho_{V}$ and $\varrho_{L}$ very close to $\varrho_{C}$, the ratio $\varrho_{V} / \varrho_{L}$ is very close to one. Hence we can use the approximation $\ln z \cong(z-1)-(z-1)^{2} / 2$, 
where $z=\varrho_{V} / \varrho_{L}$. From (8) we get

$$
\left\{\begin{array}{l}
\dot{\varrho}_{V}=\frac{\varrho_{L}-\varrho_{V}}{2\left(3\left(\varrho_{V}-\varrho_{C}\right)^{2}+T\right)}, \\
\dot{\varrho}_{L}=\frac{\varrho_{V}-\varrho_{L}}{2\left(3\left(\varrho_{L}-\varrho_{C}\right)^{2}+T\right)} .
\end{array}\right.
$$

Studying (9) we can assume $\varrho_{C}=0$. Thus

$$
\left\{\begin{array}{l}
\dot{\varrho}_{V}=\frac{\varrho_{L}-\varrho_{V}}{2\left(3 \varrho_{V}^{2}+T\right)}, \\
\dot{\varrho}_{L}=\frac{\varrho_{V}-\varrho_{L}}{2\left(3 \varrho_{L}^{2}+T\right)} .
\end{array}\right.
$$

The solution of the system (10) describes the behaviour of the curves $\varrho_{V}(T)$ and $\varrho_{L}(T)$ given by $(8)$ near the critical point $\left(\varrho_{C}, T_{C}\right)=(0,0)$.

In the next section we will consider the system of equations (10) under some conditions having a physical meaning, namely $\dot{\varrho}_{V}\left(T_{C}\right)=\infty, \quad \dot{\varrho}_{L}\left(T_{C}\right)=-\infty, \quad \frac{\partial P}{\partial \varrho}\left(\varrho_{V}(T), T\right) \neq 0, \quad \frac{\partial P}{\partial \varrho}\left(\varrho_{L}(T), T\right) \neq 0$.

We are interested in finding the unique initial conditions $\varrho_{V}\left(T_{0}\right)=\varrho_{V}^{0}$ and $\varrho_{L}\left(T_{0}\right)=\varrho_{L}^{0}$, where $\varrho_{V}^{0}<\varrho_{L}^{0}$, for which the system (10) has a solution passing through the critical point.

4. Analysis of the mathematical model. We change notation and denote the vapour density $\varrho_{V}(T)$, liquid density $\varrho_{L}(T)$ and temperature $T$ by $x(t), y(t)$ and $t$, respectively. Thus the system (10) can be written in the following form:

$$
\left\{\begin{array}{l}
\dot{x}=\frac{y-x}{2\left(3 x^{2}+t\right)}, \\
\dot{y}=\frac{x-y}{2\left(3 y^{2}+t\right)} .
\end{array}\right.
$$

We shall study the system (11) with the initial conditions $x\left(t_{0}\right)=x_{0}$ and $y\left(t_{0}\right)=y_{0}\left(x_{0}<y_{0}\right)$.

DEFINITION 1. By a solution of the system (11) we mean a pair $(x(t), y(t))$ of functions such that

(i) $x(t), y(t) \in C^{1}\left(\left[t_{0}, t^{*}\right)\right)$;

(ii) $x\left(t^{*}\right)=y\left(t^{*}\right)$ for some $t^{*}>t_{0}$;

(iii) $\left|\dot{x}\left(t_{-}^{*}\right)\right|=\left|\dot{y}\left(t_{-}^{*}\right)\right|=\infty$;

(iv) $x(t)<y(t)$ for $t \in\left[t_{0}, t^{*}\right)$.

THEOREM 2. If $(x(t), y(t))$ is a solution of (11) then

$$
-x^{2}-x y-y^{2}=t \quad \text { for } t \in\left[t_{0}, t^{*}\right] \text {. }
$$


Proof. We can transform the equations (11) to the form

$$
\begin{aligned}
& 2\left(3 x^{2}+t\right) \dot{x}=y-x, \\
& 2\left(3 y^{2}+t\right) \dot{y}=x-y .
\end{aligned}
$$

Subtracting (13) and (14) we obtain

$$
\left(3 x^{2}+t\right) \dot{x}-\left(3 y^{2}+t\right) \dot{y}=y-x .
$$

Hence we have

$$
\frac{d}{d t}\left(x^{3}+t x\right)-\frac{d}{d t}\left(y^{3}+t y\right)=0
$$

Thus

$$
x^{3}-y^{3}+t(x-y)=c, \quad \text { where } c=\text { const. }
$$

The equation (15) has to be satisfied for $t \in\left[t_{0}, t^{*}\right)$. By continuity it also holds for $t=t^{*}$.

Since $x\left(t^{*}\right)=y\left(t^{*}\right)$, we have $c=0$, and as $x(t)<y(t)$ for $t \in\left[t_{0}, t^{*}\right)$, we obtain

$$
-x^{2}-x y-y^{2}=t .
$$

By continuity (16) holds on the interval $\left[t_{0}, t^{*}\right]$. The theorem is proved.

CoRollary 3. If $x(t)$ and $y(t)$ satisfy the system (11), then the curves $x(t)$ and $y(t)$ can cross only for $t^{*}=0$.

Proof. Since the left-hand side of (12) is non-positive definite, we obtain $t_{0}<t^{*} \leq 0$. Thus the curves $x(t)$ and $y(t)$ cannot cross for $t>0$.

By (12) the system (11) can be transformed into the autonomous system

$$
\left\{\begin{array}{l}
\dot{x}=-\frac{1}{2(2 x+y)}, \\
\dot{y}=-\frac{1}{2(2 y+x)} .
\end{array}\right.
$$

Now suppose $x\left(t^{*}\right)=y\left(t^{*}\right)$, where $t^{*}<0$. Then $x^{*} \neq 0$ by (12). Thus by (17) we get $\left|\dot{x}\left(t^{*}\right)\right|=\left|\dot{y}\left(t^{*}\right)\right|<\infty$. This contradicts our definition of solution.

If $t^{*}=0$ then from (12) we have $x\left(t^{*}\right)=y\left(t^{*}\right)=0$. Moreover, by (17), $\left|\dot{x}\left(0_{-}\right)\right|=\left|\dot{y}\left(0_{-}\right)\right|=\infty$. This ends the proof of the corollary.

Theorem 4. Let $t_{0}<0$ and $\left(t^{*}, x\left(t^{*}\right)\right)=\left(t^{*}, y\left(t^{*}\right)\right)=(0,0)$. Then the system (11) has a unique solution.

Proof. Using the substitution

$$
\left\{\begin{array}{l}
x(t)=\xi(t)-\eta(t) \\
y(t)=\xi(t)+\eta(t)
\end{array}\right.
$$

we transform the condition (12) to the form 


$$
t=-\left(3 \xi^{2}+\eta^{2}\right)
$$

which is valid for $t \in\left[t_{0}, t^{*}\right]$.

By (18) and (19), from (11) we get

$$
\dot{\xi}=-\frac{3 \xi}{24 \xi^{2}+2 t} .
$$

Moreover, the function $\eta(t)$ is coupled with the solution $\xi(t)$ of $(20)$ by the condition (19). Additionally, $\xi(0)=\eta(0)=0$ by our assumption.

If $\xi \not \equiv 0$ we obtain the first order linear nonhomogeneous differential equation

$$
\frac{d t}{d \xi}+\frac{2}{3 \xi} t=-8 \xi
$$

Solving it we get

$$
t=-3 \xi^{2}+\frac{c}{\sqrt[3]{\xi^{2}}}
$$

where $c=$ const. We infer $\xi(0)=0$ if and only if $c=0$. Thus $\xi= \pm \sqrt{-t / 3}$. By (19) we also have $\eta(t) \equiv 0$, which implies $x(t) \equiv y(t)$. This contradicts the assumption that $x(t)<y(t)$.

If $\xi \equiv 0$ we obtain $\eta= \pm \sqrt{-t}$. Thus by (18) we get $x=-\sqrt{-t}$ and $y=\sqrt{-t}$ for $x(t)<y(t)$, which is the unique solution of the system (11) crossing at the critical point. The theorem is proved.

COROLlary 5. There exist unique initial conditions $x_{0}=-\sqrt{-t_{0}}$ and $y_{0}=\sqrt{-t_{0}}$, where $t_{0}<0$, for which the system (11) has a solution $(x(t), y(t))$ defining the VLE curve passing through the critical point.

5. Conclusions. We have found the unique solution of the system (11) which satisfies the conditions mentioned in Definition 1. This means that there exist unique curves $\varrho_{V}(T)$ and $\varrho_{L}(T)$ which satisfy the system $(10)$ and cross at $(0,0)$. By Theorem 4 we see that the point $\left(t^{*}, x\left(t^{*}\right)\right)=\left(t^{*}, y\left(t^{*}\right)\right)=$ $(0,0)$ agrees with the physical critical point $\left(t_{C}, \varrho_{C}\right)=(0,0)$. The unique solution of the system (11) is an approximation of the solution of the system (8) which characterizes the behaviour of the curves $\varrho_{V}$ and $\varrho_{L}$ near the critical point.

We expect that this research can be useful for further tests in the numerical determination of the phase transition curve.

\section{References}

[1] P. M. Chaikin and T. C. Lubensky, Principles of Condensed Matter Physics, Cambridge Univ. Press, Cambridge, 2000. 
[2] J. P. Hansen and I. R. McDonald, Theory of Simple Liquids, Academic Press, New York, 1976.

[3] P. Hartman, Ordinary Differential Equations, Wiley, New York, 1964.

[4] W. Okrasiński, M. I. Parra and F. Cuadros, Mathematical modeling of the VLE curve of Lennard-Jones fluids. Application to calculating the vapour pressure, Phys. Lett. A $282(2001), 36-42$.

[5] - - - - A new numerical procedure to determine the VLE curve, Computers \& Chemistry 25 (2001), 483-488.

Faculty of Mathematics, Computer Science and Econometrics

University of Zielona Góra

Szafrana 4a

65-516 Zielona Góra, Poland

E-mail: t.sulkowski@wmie.uz.zgora.pl

Received on 8.9.2006;

revised version on 21.8.2007 
